\title{
A Functional Hepatocyte Nuclear Factor 3 Binding Site Is a Critical Component of the Duck Hepatitis B Virus Major Surface Antigen Promoter
}

\author{
TRINALISA WELSHEIMER AND JOHN E. NEWBOLD* \\ Department of Microbiology and Immunology, School of Medicine, University of North Carolina at Chapel Hill, \\ Chapel Hill, North Carolina 27599-7290
}

Received 24 May 1996/Accepted 5 September 1996

\begin{abstract}
The gene coding for the $S$ protein, the smaller of the two envelope antigens of the duck hepatitis $B$ virus (DHBV), is transcribed from a TATA-less promoter. In this study, we localized the promoter to a 245-bp segment of the genome that was capable of efficiently driving expression of a linked reporter gene upon transient transfection into the differentiated hepatoma cell lines LMH and HepG2. However, no measurable activity from this construct could be detected in similar assays with the dedifferentiated cell line HepG2.1 or the nonhepatic cell line HeLa. Located at position -25 relative to the transcriptional start site was a sequence conforming to the consensus binding site for hepatocyte nuclear factor 3 (HNF3). Deletion of this region reduced activity of the reporter gene to barely detectable levels in LMH cells. The results of electrophoretic mobility shift analysis (EMSA) demonstrated that a double-stranded oligonucleotide containing this sequence formed a specific complex with DNA-binding proteins from LMH and HepG2 cells but not with nuclear extracts obtained from HepG2.1 or HeLa cells. Cotransfection of HepG2.1 cells with DHBV S promoter constructs and a rat $\mathrm{HNF3 \beta}$ expression plasmid resulted in transactivation of only those constructs in which the candidate HNF3 site was present. Furthermore, EMSA using HepG2.1 nuclear extracts containing exogenously expressed HNF3 formed complexes with the same migration and competition properties as those in which the proteins were derived from the differentiated hepatoma cells. Thus, several lines of evidence suggest a critical role for HNF3 in activity from the DHBV $S$ promoter.
\end{abstract}

Hepadnaviruses are a family of DNA-containing enveloped animal viruses for which the prototype is the hepatitis $B$ virus (HBV) of humans. Other members include the woodchuck hepatitis virus, duck HBV (DHBV), and heron HBV. These viruses display a pronounced tissue tropism, with replication occurring primarily in hepatocytes, a specificity which is thought to reflect both the presence of appropriate receptors on the surfaces of hepatic cells and the requirement for liverenriched transcription factors for the coordinate production of viral mRNAs. Indeed, diverse studies on transcription from HBV promoters have demonstrated that numerous liver-enriched proteins play roles in the regulation of at least some of these promoters $(4,5,11,14,22,23)$.

For all hepadnaviruses, the template for viral RNA transcription in productive infections is a unit-length covalently closed circular DNA molecule, initially formed from the relaxed open circular genome of the infecting virus. In DHBVinfected hepatocytes, three distinct mRNAs are synthesized from the covalently closed circular DNA template: a 3.3-kb transcript termed the pregenome, from which the nucleocapsid and polymerase polypeptides are translated, and the two envelope mRNAs of 2.3 and $2.1 \mathrm{~kb}$, from which the pre-S and $\mathrm{S}$ proteins, respectively, are produced. Though one open reading frame encodes both envelope proteins, the mRNAs from which they are translated are initiated at separate regions of the genome and thus are assumed to be under control of distinct promoters. The pre-S promoter contains a canonical TATA motif, whereas the S mRNA is not governed by a recognizable TATA sequence. Moreover, the $\mathrm{S}$ promoter is buried within the coding region for both the larger pre-S

\footnotetext{
"Corresponding author. Phone: (919) 966-5196. Fax: (919) 9628103 .
}

polypeptide and, in a different translation frame, the polymerase protein. Thus, the sequences directing transcription of the 2.1-kb mRNA must function in two alternative contexts.

Many aspects of hepadnaviral replication and biology have been dissected by using DHBV as a model; however, relatively few studies have addressed the regulation of its genes. Studies with transfected cell cultures have tentatively identified some of the cis elements involved in transcription of the mRNAs (3, 9, 17, 24); however, the transcription factors which interact with the promoters have not been extensively characterized. A region upstream of the DHBV nucleocapsid promoter has been described as a liver-specific enhancer $(9,17,24)$, and liver-enriched transcription factors have been demonstrated to recognize binding sites within the enhancer region $(16,18)$. Only one of these studies (17) examined the effects of the enhancer on the DHBV promoters. That report concluded that the enhancer augmented the activity of a DHBV minimal nucleocapsid promoter construct and had no effect on activity from the pre-S promoter but was essential for expression from the $\mathrm{S}$ promoter.

Whereas gene regulation studies of the DHBV system are in their infancy, numerous studies have investigated the sequences responsible for regulation of transcription from the HBV promoters $(4,5,11,14,21-23,25)$. Diverse studies have indicated that the HBV $\mathrm{S}$ promoter is active in both liverderived and non-liver cell lines $(5,11,22)$. Consistent with these observations is the important role played by Sp1, a ubiquitously expressed transcription factor, in regulating activity from this promoter (21). In contrast, transcription from the DHBV S promoter has been described as liver specific, a preference which has been attributed to the dependence of the DHBV S promoter on the viral enhancer (17).

The work presented here describes the identification of a 
key regulatory sequence in the DHBV $\mathrm{S}$ promoter. We have employed deletion analysis of a unit-length copy of the DHBV genome in transient-expression experiments to examine the expression of a linked reporter gene from the DHBV S promoter. We found that while the DHBV S promoter displayed a requirement for differentiated hepatocytes, the DHBV enhancer was not necessary for expression from the $\mathrm{S}$ promoter in our assay system. We observed that a DHBV fragment of 245 bp (positions -50 to +195 relative to the transcription start site) was necessary and sufficient for high levels of expression of a reporter gene in transient transfections into LMH or HepG2 cells. We present evidence that a sequence within this fragment binds members of the hepatocyte nuclear factor 3 (HNF3) family of liver-enriched proteins and that this binding site represents a critical component of the DHBV S promoter.

\section{MATERIALS AND METHODS}

Plasmid constructions. The steps used for construction of the various plasmids used in the transient-transfection experiments followed standard molecular biology protocols (1). The DHBV sequences in these constructions were derived from pBR322-p2.3 (GenBank accession no. M60677), which contains a head-totail dimer of the viral DNA sequence inserted at the EcoRI site of pBR322. The chloramphenicol acetyltransferase (CAT) reporter plasmid was the promoterless pCATBasic vector (Promega). The plasmid pBS was created by digestion of pBR322-p2.3 at a unique DHBV XhoI site and cloning the unit-length genomic fragment into the SalI site of pCATBasic. The unique XhoI site is situated between the reported cap site for the $S$ transcript at nucleotide 984 and the methionine codon at nucleotide 1284 (3). Therefore, pBS contains the complete DHBV genome (coordinates 1213 to 3021 and 1 to 1217) permuted such that transcription of the CAT gene is controlled by the DHBV S promoter. Deletions from the $5^{\prime}$ end of the DHBV genome in pBS were generated by excising selected sequences between restriction endonuclease sites. Where this was not feasible, PCR was used to engineer appropriate restriction sites at the ends of desired DHBV fragments, followed by cloning steps similar to those used for pBS. Mutation of the pre-S TATA box (see Fig. 1B) was achieved by a two-step overlapping PCR method, in which the TATA sequence at DHBV nucleotide coordinates 710 to 715 , TATAAG, was first altered to a PstI site, CTGCAG, via two half reactions and 10 cycles of amplification, followed by mixing of the products and a subsequent 10 cycles of amplification. The incorporation of the PstI restriction site provided a convenient means for screening plasmid DNA following bacterial transformation, as there are no native Pst I sites in the DHBV p2.3 genome. Plasmids are designated according to DHBV sequences present in the construct, using coordinates derived from the GenBank sequence data bank. All plasmids were purified through two isopycnic centrifugations in $\mathrm{CsCl}$ with ethidium bromide.

Cells and transfections. The chicken hepatoma cell line $\operatorname{LMH}(6,15)$ and the human hepatoma cell lines HepG2 and HepG2.1 (22) were grown in Dulbecco's modified Eagle's medium-Ham's F-12 medium (1:1), supplemented with $10 \%$ fetal bovine serum, at $37^{\circ} \mathrm{C}$ in humidified $5 \% \mathrm{CO}_{2}$. The human cervical carcinoma cell line HeLa was grown in Dulbecco's modified Eagle's medium plus 4.5 $\mathrm{mg}$ of glucose per $\mathrm{ml}$ and $10 \%$ fetal bovine serum at $37^{\circ} \mathrm{C}$ in $5 \%$ humidified $\mathrm{CO}_{2}$.

LMH, HepG2.1, and HeLa cells were transfected by electroporation (1). Exponentially growing cells were harvested, washed twice with ice-cold phosphate-buffered saline (PBS), and suspended to $2 \times 10^{7}$ cells per $\mathrm{ml}$ in ice-cold PBS. The cells were electroporated at $960 \mu \mathrm{F}$ and $250 \mathrm{~V}$, using a Bio-Rad Gene Pulser. HepG2 cells were transfected by the calcium phosphate coprecipitation technique as described previously (1). For both methods, the transfected DNA mixture consisted of $5 \mu \mathrm{g}$ of a CAT plasmid and $1 \mu \mathrm{g}$ of pCMV $\beta$ (Clontech), an internal control for transfection efficiency. $\mathrm{pCMV} \beta$ harbors the $\beta$-galactosidase gene of Escherichia coli, under the control of the cytomegalovirus immediateearly promoter. In certain experiments, the DNA mixture also included $1.5 \mu \mathrm{g}$ of the HNF3 $\alpha$ or HNF3 $\beta$ expression vector, pCMVHNF3 $\alpha$ or pCMVHNF3 $\beta$, respectively, or an equivalent amount of empty expression vector pCMV as a control. Cell extracts were prepared 21 to $24 \mathrm{~h}$ posttransfection and assayed for CAT activity by ascending thin-layer chromatography (1). Chromatograms were quantitatively scanned by using a radioanalytic imaging system (AMBIS). An aliquot of each extract was also assayed for $\beta$-galactosidase activity by using a kit from Promega. Within a transfection experiment, the CAT activity of each S promoter construct was normalized (percent acetylation per unit of $\beta$-galactosidase activity) and then compared with the normalized activity of a reference $S$ promoter construct. The resulting ratio was the relative CAT activity for that construct. For LMH and HepG2 cells, the reference point was the unit-length genomic construct $\mathrm{pBS}$. In LMH cells, acetylation values for $\mathrm{pBS}$ were generally between 3 and $7 \%$ after subtraction of background acetylation from the empty pCATBasic vector. All transient-expression assays were performed 2 to 12 times, using at least two independent plasmid preparations.

Nuclear extracts and electrophoretic mobility shift assays. Nuclear extracts from LMH, HepG2, and HepG2.1 cells were prepared by using a modified rapid-lysis protocol (2). All procedures were performed at 0 to $4^{\circ} \mathrm{C}$ with ice-cold reagents. Cells were scraped into PBS, pelleted, washed twice with PBS, and resuspended in lysis buffer $\left(10 \mathrm{mM} N\right.$-2-hydroxyethylpiperazine- $N^{\prime}$-2-ethanesulfonic acid [HEPES] [pH 7.6], $60 \mathrm{mM} \mathrm{KCl}, 1 \mathrm{mM}$ EDTA, $1 \mathrm{mM}$ dithiothreitol, protease inhibitors [2], $0.1 \%$ Nonidet P-40). After a 4-min incubation on ice, the lysates were spun for $4 \mathrm{~min}$ at $1,500 \mathrm{rpm}$ in an Eppendorf microcentrifuge at $4^{\circ} \mathrm{C}$ and the supernatants were removed. The pelleted nuclei were resuspended in lysis buffer without Nonidet P-40, pelleted by centrifugation at $1,500 \mathrm{rpm}$ for 4 min, then resuspended in nuclear extract buffer $(20 \mathrm{mM}$ Tris hydrochloride $[\mathrm{pH}$ 8.0 ], $420 \mathrm{mM} \mathrm{NaCl}, 1.5 \mathrm{mM} \mathrm{MgCl}, 0.2 \mathrm{mM}$ EDTA, $1 \mathrm{mM}$ dithiothreitol, $25 \%$ [vol/vol] glycerol, protease inhibitors), and incubated on ice for $10 \mathrm{~min}$ with occasional vortexing. The suspension was pelleted at $4^{\circ} \mathrm{C}$ in an Eppendorf a microcentrifuge at $15,000 \mathrm{rpm}$ for $10 \mathrm{~min}$, and the supernatant was removed and dialyzed for $2 \mathrm{~h}$ at $4^{\circ} \mathrm{C}$ against dialysis buffer (20 mM HEPES [pH 7.9], $75 \mathrm{mM}$ $\mathrm{KCl}, 0.2 \mathrm{mM}$ EDTA, $10 \%$ [vol/vol] glycerol, protease inhibitors). The dialysate was pelleted briefly at $4^{\circ} \mathrm{C}$, and the supernatant was removed, aliquoted, and stored at $-70^{\circ} \mathrm{C}$. Protein concentration was determined by the Bradford assay (Bio-Rad) against a bovine serum albumin standard. HeLa cell extracts were purchased from Promega and used as directed. For preparation of extracts containing exogenously expressed $\mathrm{HNF} 3 \alpha$ or HNF3 $\beta$, HepG2.1 cells were electroporated with $15 \mu \mathrm{g}$ of the expression vector $24 \mathrm{~h}$ prior to extract preparation.

Electrophoretic mobility shift assays (EMSAs) were carried out essentially as described elsewhere (1). ${ }^{32} \mathrm{P}$-labeled double-stranded oligonucleotide (see below) $\left(6 \times 10^{-2} \mathrm{pmol}\right)$ was incubated with $4 \mu \mathrm{g}$ of nuclear extract prior to $4 \%$ polyacrylamide gel electrophoresis at $4^{\circ} \mathrm{C}$ and autoradiography. When competition experiments were performed, nuclear extract was preincubated with 9 pmol of unlabeled double-stranded competitor oligonucleotide for $10 \mathrm{~min}$ prior to the addition of the labeled oligonucleotide.

Oligonucleotides and probes. The double-stranded oligonucleotides used were synthesized as complementary single strands that spanned selected DHBV sequences or comprised previously identified consensus binding sites (see Fig. 3). In addition to the sequences shown, double-stranded oligonucleotides contained $5^{\prime}$ AGCT extensions, which were labeled by filling in with $\left[\alpha-{ }^{32} \mathrm{P}\right] \mathrm{dATP}$, unlabeled deoxyribonucleoside triphosphates, and the Klenow fragment of DNA polymerase.

\section{RESULTS}

Deletion analysis of the $\mathbf{S}$ promoter. Evaluation of the regulatory regions governing transcription of the DHBV surface antigen promoter began with a full-length copy of the DHBV genome inserted such that the S promoter was immediately adjacent to and upstream of the CAT gene. The abilities of constructs with a series of $5^{\prime}$ deletions of the DHBV sequence to direct transcription of the reporter gene were compared, using transient transfection assays in a variety of cell lines (Fig. 1A). In the chicken hepatoma cell line LMH and the human hepatoblastoma cell line HepG2, removal of as much as $84 \%$ of the DHBV genome from the parent construct did not have an adverse effect on transcription of the reporter gene. In fact, removal of sequences $5^{\prime}$ to DHBV nucleotide coordinate 428 resulted in consistent and marked increases in transcriptional activity compared with that of $\mathrm{pBS}$, as indicated by the increased relative CAT values. The only drop in signal occurred upon deletion of sequences between positions 428 and 720 . This 2.7-fold reduction may reflect the removal of supplemental elements modulating the $\mathrm{S}$ promoter. Nevertheless, since the activity of pB720-1217 was at least as strong as the genomic construct in the differentiated liver cell lines, we concluded that basal requirements for efficient transcription from the $\mathrm{S}$ promoter were represented in pB720-1217. None of the constructs exhibited detectable activity in the dedifferentiated HepG2.1 or the non-liver HeLa cell line, even when the amount of transfected construct was increased to $15 \mu \mathrm{g}$ (data not shown), though expression from positive-control plasmids, such as pRSVCAT and pCMV $\beta$, indicated that the lack of signal was not due to problems with the transfection technique.

In addition to the $\mathrm{S}$ promoter, pB428-1217 includes the primary sequences responsible for directing transcription from the pre-S promoter $(3,17$; unpublished observation), including the pre-S TATA motif. The $5^{\prime}$ breakpoint for $\mathrm{pB} 720-1217$ lies immediately downstream of this sequence. Thus, another in- 


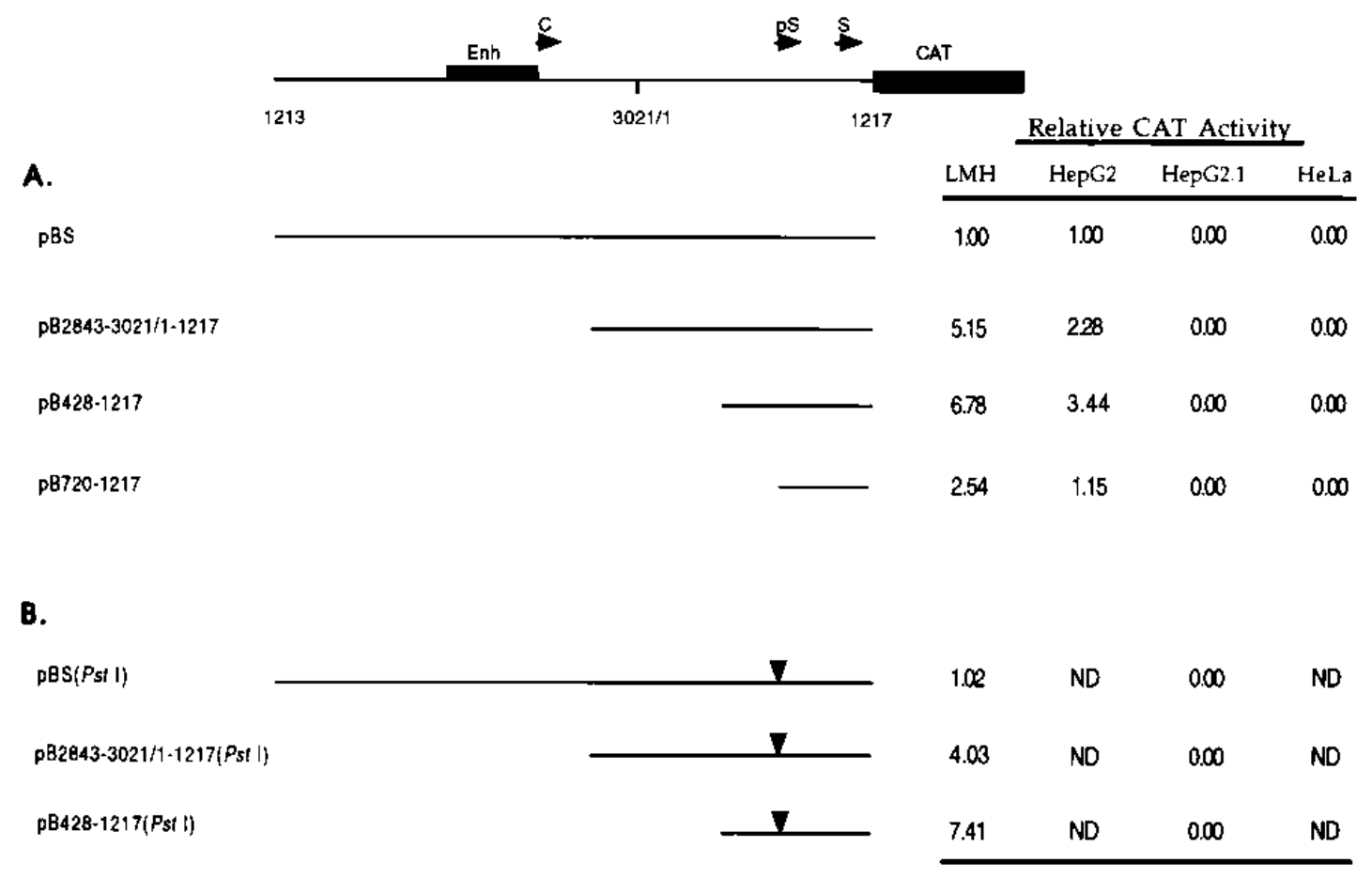

FIG. 1. Deletion analysis of the DHBV S promoter. The diagram at the top of the figure indicates the orientation of the unit-length DHBV genome permuted so that the S promoter directs transcription of the CAT gene. Arrows represent the positions and directions of transcription from the pregenome-nucleocapsid (C), presurface (pS), and surface (S) promoters. Black boxes indicate the enhancer (Enh) and the CAT open reading frame. The activity of each of the various promoter constructs is reported relative to the activity of the pBS construct, which is set at 1.00. (A) Deletion analysis of the S promoter in LMH, HepG2, HepG2.1, and HeLa cells. The horizontal lines indicate the DHBV sequences present in the various S promoter constructs. Plasmids are designated according to the DHBV sequences present in each construct, using nucleotide coordinates derived from the GenBank genetic sequence data bank. (B) Relative activities of the S promoter deletion constructs in the context of the pre-S TATA box mutation. Inverted arrowheads indicate the locations of the substitution of the PstI site for the TATA box. Plasmids are designated as described above, with "(PstI)" included to indicate the presence of the mutation. Activities of the mutated plasmid constructs are reported relative to that of pBS. ND, not done.

terpretation for the increased expression from pB428-1217 could be a positive influence of the pre-S promoter in the form of read-through into the reporter gene, producing an additive effect on CAT expression. Consistent with this notion was the decreased signal from the reporter gene upon deletion of DHBV sequence between coordinates 428 and 720 , including the TATA box sequence at coordinates 710 to 715 . Though the presence of multiple ATG codons between the pre-S mRNA cap site at DHBV nucleotide 732 and the CAT protein ATG more than 400 nucleotides downstream made this possibility seem unlikely, we nevertheless wished to assess the activity of the $\mathrm{S}$ promoter in the presence of an inactive pre-S promoter. In previous experiments with the pre-S promoter, we had observed that mutation of the TATA sequence to a PstI site ablated expression from pre-S promoter constructs (unpublished observation). Thus, we made a series of constructs analogous to those shown in Fig. 1A but with the pre-S TATA box replaced by a PstI site and monitored their activities in LMH cells (Fig. 1B). Expression levels of the altered constructs were not significantly different from those of the unmutated counterparts; furthermore, they exhibited the same trend in activity with successive deletions from the $5^{\prime}$ end. These observations indicated that transcription from the pre-S promoter was not being detected by the transient-expression assays and that the pre-S promoter was neither a positive nor negative influence on the transcriptional activity of the $\mathrm{S}$ promoter in this system.

The experiments described above demonstrated that pB7201217 possessed the necessary components to direct transcription from the $\mathrm{S}$ promoter in a cell type-specific manner. Ad- justment to the $3^{\prime}$ end of this construct was made by deletion of sequence between DHBV nucleotide coordinates 1179 and 1217; this alteration did not adversely affect reporter gene activity in LMH cells (Fig. 2). An additional 5' deletion beyond the transcription start site at DHBV nucleotide coordinate 984 (3) resulted in loss of signal from the reporter gene (pB10941178 [Fig. 2]), so we concluded that a functional DHBV S promoter lay between nucleotide coordinates 720 and 1178 .

Identification of a sequence upstream of the $S$ mRNA initiation site with properties of an $\mathrm{HNF3}$ recognition site. Since

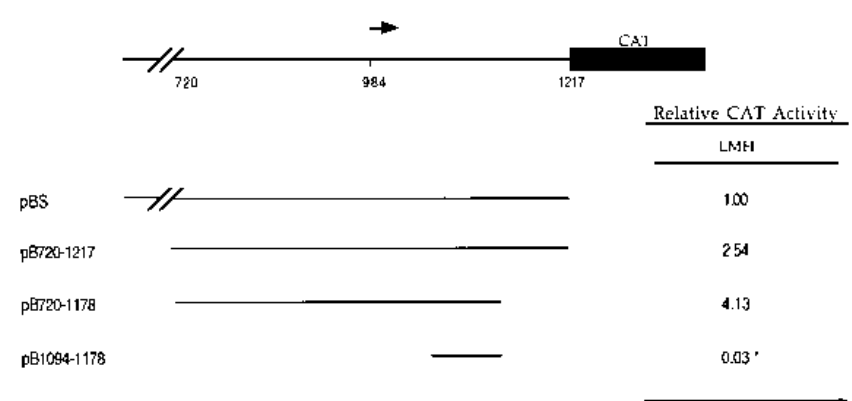

FIG. 2. Deletion analysis of pB720-1217 in LMH cells. The diagram at the top represents pBS with the region downstream of nucleotide coordinate 720 enlarged. The arrow indicates the transcriptional start site at DHBV coordinate 984. Horizontal lines and plasmid designations are as described in the legend to Fig. 1. The asterisk indicates that activity from this construct could not always be distinguished from the background activity of the pCATBasic plasmid. 


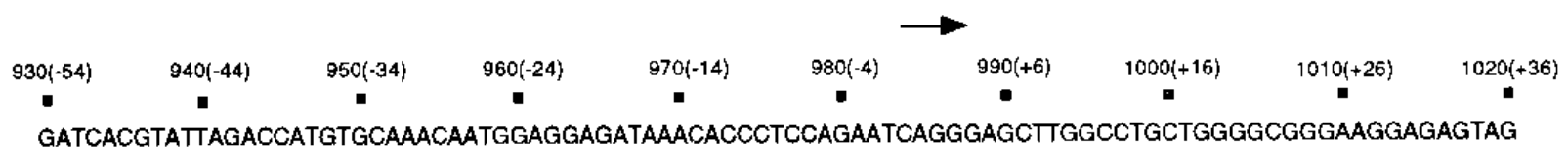

\author{
DHBV oligo:

$\begin{array}{ll}\text { Consensus oligo: } & \\ \text { TTR (HNF3) } & \text { CTGATTCTGATATTGACTAGTCA } \\ \text { Sp } 1 & \text { GATCGGGGCGGGGCGAGC }\end{array}$

FIG. 3. Nucleotide sequence of the DHBV S promoter region. The nucleotide coordinates are derived from the GenBank database, and their positions relative to the transcription initiation site at nucleotide 984 (indicated with an arrow) are shown in parentheses. The sequences present in the DHBV oligonucleotides (DHBV oligo) (top strand only) are indicated and are designated according to the coordinates of the DHBV sequences present. Sequences of the oligonucleotides representing consensus binding sites are shown boxed. TTR (HNF3) is a previously characterized HNF3 binding site from positions -85 to -111 of the mouse transthyretin promoter. Sp1 is the consensus recognition sequence for the Sp1 transcription factor. The binding site within each of those oligonucleotides is underlined. The HNF3 consensus recognition sequence is aligned with the DHBV S promoter candidate HNF3 binding site at positions -25 to -36 , and identical nucleotides are indicated ( $\mathrm{K}$ is $\mathrm{G}$ or $\mathrm{T}$; R is A or G; V is A, C, or G; W is A or T; and Y is C or T). Also shown is an alignment between the HNF3 consensus recognition sequence and the sequence from DHBV oligonucleotide 961-983 around the TGTTT core.

the region immediately upstream of the initiation site for $\mathrm{S}$ was fully capable of activating transcription and imparting liver specificity in transient-expression assays, this region was examined for sequences that displayed similarities to known hepatocyte-enriched regulatory proteins. A striking match to the HNF3 consensus recognition site (20) was located on the DHBV antisense strand, at positions -25 to -36 (nucleotide coordinates 948 to 959) relative to the mRNA initiation site for $\mathrm{S}$ at nucleotide 984 (Fig. 3). EMSAs were conducted to evaluate the affinity and specificity of this sequence for DNAbinding proteins contained in nuclear extracts from LMH cells. The double-stranded oligonucleotides used in these assays are shown in Fig. 3.

In EMSA, proteins extracted from LMH nuclei specifically bound the $945-959$ probe, as demonstrated by the addition of unlabeled double-stranded oligonucleotide $945-959$ or unlabeled double-stranded TTR oligonucleotide (Fig. 4, lanes 2 to 4), which contained a known high-affinity HNF3 binding site from the transthyretin promoter (7). Addition of an unlabeled double-stranded oligonucleotide specific for Sp1 failed to inhibit the formation of complexes (lane 5).

To determine the extents to which the DNA-protein interactions in Fig. 4 were characteristic of differentiated liver cell lines, we used the labeled 945-959 oligonucleotide with extracts prepared from the cell lines used in the transient-expression assays (Fig. 5). LMH and HepG2 nuclear extracts displayed similar specific interactions with the $945-959$ probe (lanes 2 to $5)$, whereas these specific complexes were not detectable in reactions with HepG2.1 or HeLa extracts. HeLa extracts occasionally produced a low level of a complex which migrated more slowly than those formed with LMH or HepG2 extracts; however, this complex was inconsistently inhibited by the addition of unlabeled 945-959 oligonucleotide. The apparent liver-enriched distribution of the proteins interacting with probe 945-959 together with the demonstrated specificity of the DNA-protein interactions supported the contention that proteins with properties similar to HNF3 were capable of recognizing this DHBV sequence in these assays.
Deletion of the putative $\mathrm{HNF3}$ recognition sequence drastically reduces expression from the $S$ promoter. Sequence inspection and EMSAs indicated that the identified sequence might be a binding site for HNF3. However, those experiments did not address the functional contribution of the site in the context of the $\mathrm{S}$ promoter, and such a determination could not be made from the constructs used for the earlier transienttransfection experiments. Therefore, this region was further analyzed with additional 5' deletions of pB720-1178 (Fig. 6).

Deletion of sequence directly upstream of the potential

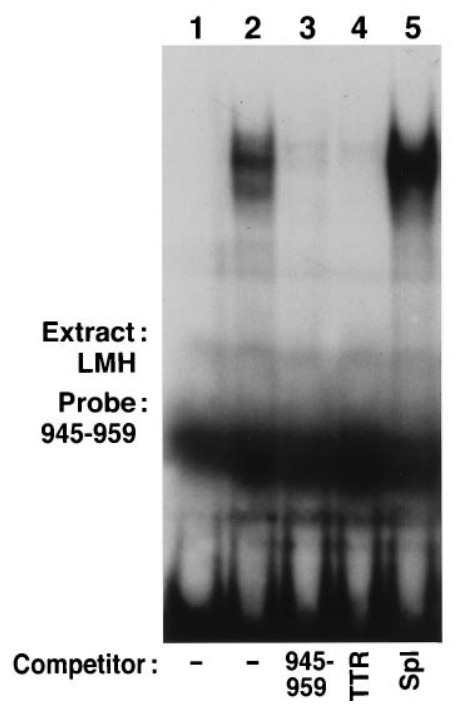

FIG. 4. EMSA and complex inhibition analysis of the radiolabeled doublestranded oligonucleotide 945-959 from the DHBV S promoter. LMH nuclear extracts were analyzed for their ability to form complexes with probe 945-959, and unlabeled double-stranded oligonucleotides were used as competitor DNAs to demonstrate the specificity of the observed complexes. Lane 1, no nuclear extract and no competitor (control); lane 2, no competitor (control). 


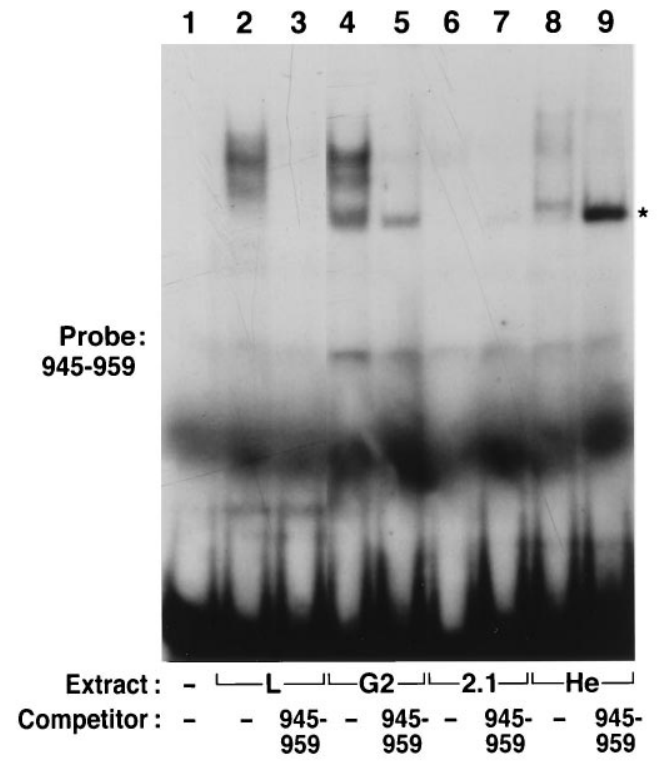

FIG. 5. DNA-protein complexes formed with extracts from different cell lines (L, LMH; G2, HepG2; 2.1, HepG2.1; He, HeLa) and radiolabeled 945-959. Unlabeled double-stranded oligonucleotide $945-959$ was used to demonstrate the specificity of the observed complexes. Extract was omitted from lane $1(-)$. The asterisk indicates the position of a frequently seen nonspecific band visible in lanes $4,5,8$, and 9 .

HNF3 site to produce pB934-1178 did not significantly affect reporter gene activity in $\mathrm{LMH}$ cells, which indicated that the signal registered from pB720-1178 was primarily dependent upon sequences downstream of nucleotide 934. In contrast, CAT expression from $\mathrm{pB} 957-1178$ was reduced more than 40fold from that of pB934-1178. Since the deletion of sequence between DHBV coordinates 720 and 934 had no significant effect on reporter gene activity, whereas the subsequent deletion to coordinate 957 produced a severe effect, we concluded that pB934-1178 represented a minimal S promoter in these

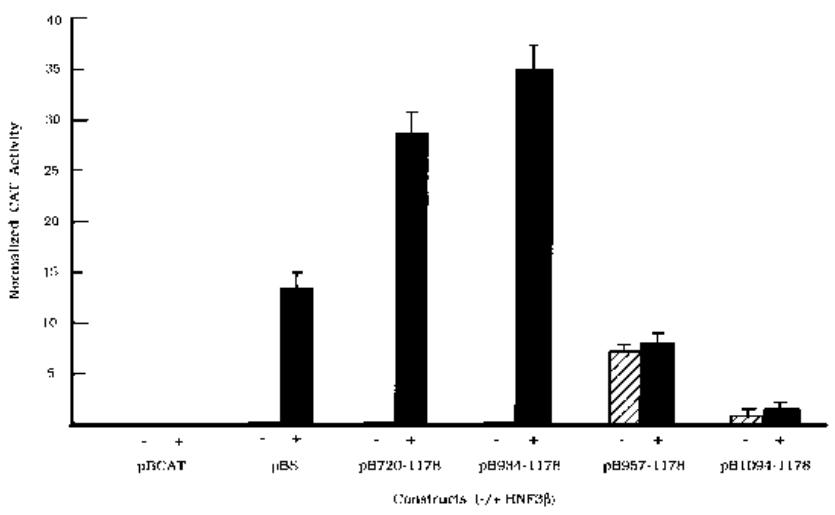

FIG. 7. Exogenous HNF3 $\beta$ activates the DHBV S promoter in HepG2.1 cells. Activities of the DHBV S promoter constructs are reported in the absence $(-)$ or presence $(+)$ of expressed HNF3 $\beta$. Since the DHBV S promoter constructs were inactive in this cell line in the absence of expressed HNF3 $\beta$, CAT activities in the presence of the expressed protein are not reported as fold transactivation. Instead, the normalized CAT activities of each of the S promoter plasmids in the presence of HNF3 $\beta$ are depicted as the average percent acetylation obtained from three experiments.

transient-expression assays and that the region between coordinates 934 to 957 spanned a significant transcriptional regulatory element.

Exogenous expression of HNF3 turns on the S promoter in HepG2.1 cells. The preceding data strongly suggested an essential role for the region between coordinates 934 and 957 in transcription from the DHBV S promoter, and EMSA results indicated that this sequence might harbor a binding site for members of the HNF3 family. To assess the functional response of the DHBV S promoter to HNF3 transcription factors, we transfected HepG2.1 cells with a rat HNF3 $\beta$ expression plasmid in concert with several $\mathrm{S}$ promoter constructs (Fig. 7). The effect of supplying HNF3 $\beta$ in trans was profound. In all cases in which the putative HNF3 recognition sequence was present, HNF3 $\beta$ was capable of activating the reporter

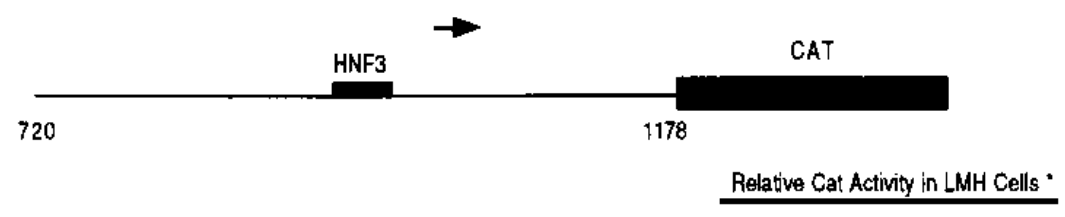

pB934-1178

pB957-1178

pB1094-1178
5.42

0.03

calculated against a value of 1,00 for pes

FIG. 6. Deletion analysis of pB720-1178 in LMH cells. The diagram at the top of the figure represents construct pB720-1178. The black boxes represent the positions of the HNF3 binding site and the CAT open reading frame. The arrow indicates the site of transcription initiation at DHBV coordinate 984. Horizontal lines and plasmid designations are as described in the legend to Fig. 1. 


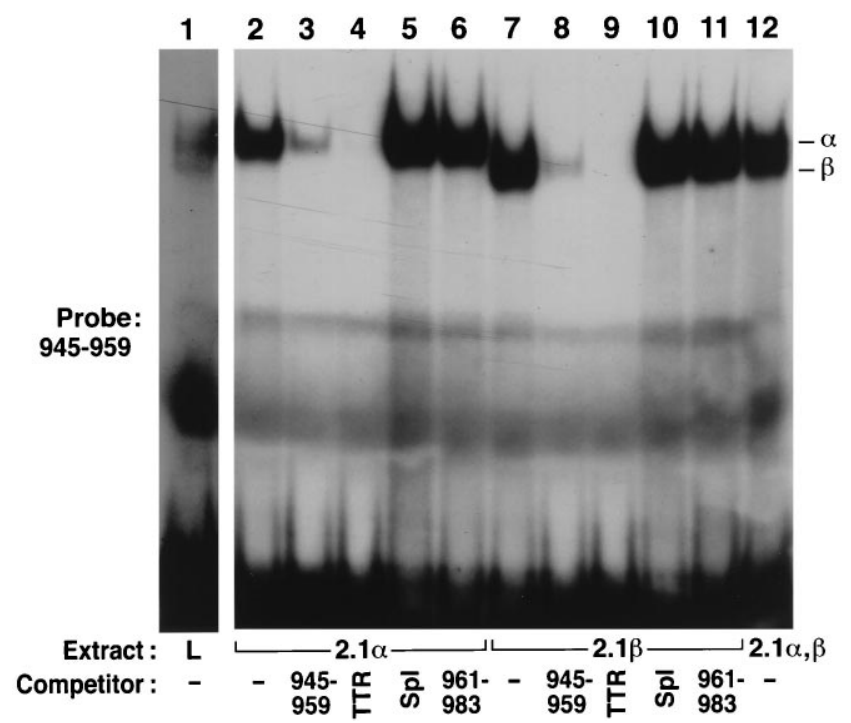

FIG. 8. Interaction of exogenously expressed HNF3 $\alpha(\alpha)$ and HNF3 $\beta(\beta)$ proteins with radiolabeled double-stranded oligonucleotide 945-959 in EMSA. Unlabeled double-stranded DNAs were used to determine the specificity of the observed complexes. Extract abbreviations: L, LMH; $2.1 \alpha$, HepG2.1 cells transfected with pCMVHNF3 $\alpha ; 2.1 \beta$, HepG2.1 cells transfected with pCMVHNF3 $\beta$; $2.1 \alpha, \beta$, equal amounts of $2.1 \alpha$ and $2.1 \beta$ extract mixed in the same binding reaction mixture. Lane 1 is from a longer exposure of the same gel. Competitor was not present $(-)$ in some lanes, as indicated below the gels.

gene, with normalized CAT activities ranging from 13 to $35 \%$. Since HepG2.1 cells previously were not capable of supporting transcription from these promoter constructs and did not do so in the presence of the pCMV vector control (Fig. 7), it appeared that the presence of HNF3 $\beta$ was a critical factor in switching these DHBV S promoter constructs from an inactive to an active state. The two constructs lacking the candidate HNF3 site (pB957-1178 and pB1094-1178) appeared to possess some level of independent activity in this cell line, but they did not exhibit any responsiveness to HNF3 $\beta$. The inability of HNF3 $\beta$ to mediate induction of the reporter gene when sequences upstream of coordinate 957 were absent implies that a functional site was no longer available in those plasmids.

Exogenously expressed HNF3 shifts the $945-959$ probe in a manner identical to that of liver cell nuclear extracts. Previously, we determined that the $945-959$ probe bound nuclear proteins in a cell type-specific manner (Fig. 4 and 5), corresponding to the expression profile observed in transient-transfection experiments (Fig. 1). Cotransfection of HepG2.1 cells with a rat HNF3 $\beta$ expression plasmid and S promoter constructs resulted in transactivation of the $S$ promoter, apparently through the identified HNF3 motif (Fig. 7). We used nuclear extracts derived from HepG2.1 cells transfected with vectors expressing rat $\mathrm{HNF} 3 \alpha$ or rat $\mathrm{HNF} 3 \beta$ in conjunction with the labeled double-stranded oligonucleotide 945-959 in EMSA to confirm the ability of these expressed proteins to interact with this sequence (Fig. 8).

Expression of either $\mathrm{HNF} 3 \alpha$ or $\mathrm{HNF} 3 \beta$ resulted in the formation of complexes with migration and competition properties similar to those seen with extracts from LMH nuclei. These complexes were not observed when extracts from HepG2.1 cells transfected with the empty expression vector pCMV were used (data not shown). Since the HNF3 proteins exist in liver cells as a family of closely related polypeptides with slightly different migration properties (20), these proteins frequently appear as an elongated complex rather than a discrete band in these types of assays. This probably accounts for exogenous expression of either one of the proteins resulting in a more distinct band than a complex formed with extracts from liver nuclei. Unlabeled competitor oligonucleotides 945-959 or TTR inhibited formation of the complexes (Fig. 8, lanes 3, 4, 8, and 9), though a small fraction of complex remained when competitor oligonucleotide 945-959 was used (lanes 3 and 8). This result is consistent with reports that have indicated that there are both strong and weak affinity sites for HNF3 proteins (20) and that recognition sequences containing a TGTTT core are weak affinity sites $(13,20)$. The addition of either the unlabeled double-stranded Sp1 consensus oligonucleotide or the DHBV double-stranded 961-983 oligonucleotide had no effect (lanes $5,6,10$, and 11). The inability of the latter oligonucleotide to compete was interesting, as this oligonucleotide contained a motif with a match at 9 of 12 positions to the HNF3 consensus sequence, including a TGTTT core identical to the one contained within double-stranded oligonucleotide 945-959 (Fig. 3 ). The inability of the 961-983 oligonucleotide to inhibit the formation of complexes despite sequence similarity was a further, albeit circumstantial, indication of the specific nature of the interaction taking place between probe 945-959 and HNF3 proteins. Though complexes between LMH nuclear extracts and labeled double-stranded oligonucleotide 961-983 could be resolved during EMSA, these complexes did not comigrate with the gels shown in Fig. 4, 5, or 8, and they were not inhibited by the addition of unlabeled double-stranded oligonucleotide 945-959 (data not shown). Use of equal amounts of HNF3 $\alpha$ and HNF3 $\beta$ together in a binding reaction with probe 945-959 produced a complex with an appearance and migration similar to the one formed by LMH nuclear extracts (Fig. 8 , lane 12). Inhibition of the formation of this complex was the same as in the assays using the individual polypeptides (data not shown).

\section{DISCUSSION}

We present several lines of evidence indicating that HNF3like proteins play a critical role in transcription from the DHBV S promoter and that this family of liver-enriched transcription factors probably mediate their regulatory effects through a binding site at positions -25 to -36 relative to the cap site for the $\mathrm{S}$ transcript. The EMSA results demonstrated the following. (i) An oligonucleotide containing the candidate binding site specifically interacted with factors from the nuclei of LMH and HepG2 cells but not those from HepG2.1 or HeLa cells. (ii) The DNA-protein complexes formed with nuclear extracts from LMH and HepG2 cells comigrated. (iii) Exogenously expressed rat HNF3 proteins formed specific complexes with this oligonucleotide that comigrated with complexes seen when LMH or HepG2 nuclei were used. Deletion of the predicted HNF3 binding site from a minimal DHBV S promoter construct caused a precipitous reduction in expression of a linked reporter gene in LMH cells. Furthermore, cotransfection of HepG2.1 cells with HNF3 expression plasmids and DHBV S promoter constructs demonstrated that the $\mathrm{S}$ promoter constructs which previously had been silent in this cell line could be activated by HNF3 but only in cases where the proposed HNF3 site was present. These data accord well with our initial observation that expression from the $\mathrm{S}$ promoter was dependent upon the environment of a differentiated hepatocyte. Formal proof that HNF3 is acting at the identified site is lacking, since antisera that would positively identify HNF3 proteins from chicken liver nuclear extracts are not available. However, the simplest explanation for our findings is 
that an HNF3-like protein(s) is an important determinant in regulating transcription from the DHBV S promoter.

Our observation that transcriptional activity from the DHBV S promoter was retained in the absence of the DHBV enhancer region at nucleotide coordinates 2172 to $2355(9,17)$ differs significantly from a previous report (17). In that analysis, inclusion of the enhancer segment was essential to detection of activity from the $\mathrm{S}$ promoter in transient transfections into LMH cells. In the current study, removal of the enhancer region from the unit-length genomic construct did not diminish transcriptional activity in LMH or HepG2 cells, which suggests that perhaps other regions of the genome are more important in regulating transcription from the $\mathrm{S}$ promoter. The reason for such different results is not clear. The extents of the DHBV sequences present in the plasmid constructs used are not likely to account for the difference, as the DHBV sequences represented in our construct pB720-1217 are analogous to those in pSGH (17), in which DHBV sequence from coordinates 718 to 1217 drives a human growth hormone reporter gene. DHBV sequences in $\mathrm{pSGH}$ were derived from the DHBV16 sequence (19). The DHBV16 genome differs from that of DHBV2.3 at 5 nucleotide positions between coordinates 718 and 1217 . Though these changes are scattered over the 500-bp region and none of them fall within the HNF3 recognition sequence identified in the present study, it is nonetheless possible that the difference in the two sequences accounts for the contrasting outcomes. Alternatively, the difference in results may be a consequence of the different reporter systems employed.

We noted consistently elevated levels of reporter gene activity relative to that of the parent plasmid construct $\mathrm{pBS}$ for the $\mathrm{S}$ promoter constructs in which the enhancer, the pregenome-nucleocapsid promoter, and significant portions of pregenome-encoding sequence were absent (Fig. 1A). Rather than reflecting specific positive regulatory elements acting on the DHBV S promoter, these data most likely result from alleviating promoter occlusion, in which transcription from downstream promoters is suppressed by strong upstream promoters (10). Consistent with this interpretation are the observations of Huang and Summers (12) during characterization of the DHBV pet sequence. They found that when the pregenome promoter was deleted or inactivated by removal of pet, there were corresponding increases in the levels of mRNA from the downstream envelope promoters, as measured by Northern (RNA) blot analysis and primer extension.

A mutation which incapacitates expression from the DHBV pre-S promoter had no effect on the amount of reporter gene expression directed by the downstream DHBV S promoter. This was an important determination for two reasons. First, the two promoters are close enough to one another to make promoter occlusion a possibility in this case as well. The fact that it is possible to inactivate the upstream promoter, in this case pre-S, and not observe a corresponding increase in activity from the downstream promoter indicates that transcriptional interference from pre-S is not occurring in this system. A similar result has been noted previously (12). Second, since expression from $\mathrm{pB} 428-1217$ did not differ from $\mathrm{pB} 428$ 1217(PstI) (Fig. 1), we can conclude that the decrease in CAT expression when sequence between coordinates 428 and 720 was deleted probably reflects removal of one or more elements that modulate the activity of the minimal $\mathrm{S}$ promoter. If the elevated activity of pB428-1217 had been a consequence of transcriptional read-through from the pre-S promoter instead, we would have expected that expression from pB4281217(Pst I) be decreased in comparison. Thus, it appears that though the fundamental components of the DHBV S promoter are in the immediate proximity of the cap site for the $S$ tran- script, additional modulatory sequences may exist in the vicinity of the DHBV pre-S promoter.

HNF3 proteins have been shown to be important regulators in the liver-specific transcription of numerous genes expressed mainly in hepatocytes (26) and in fact have recently been reported to modulate the pre-S1 and nucleocapsid promoters of HBV in transient-expression assays $(14,23)$. Several binding sites for HNF3 have been identified in the DHBV enhancer as well $(8,16,18)$. In our transactivation experiments, the effect of exogenous HNF3 $\beta$ on pBS is not as strong as its effect on pB934-1178. In the context of the entire DHBV genome, the exogenously expressed protein may be interacting with HNF3 recognition sequences present elsewhere in the DHBV sequence, though whether that would contribute to activation of other DHBV promoters is not known. The lower induction of pBS relative to those of the subsequent deletion constructs mimics the general trend we observed with the $\mathrm{S}$ promoter deletion constructs in the LMH and HepG2 cell lines. The fact that induced activity did not decrease in going from $\mathrm{pBS}$ to pB720-1178 to pB934-1178, together with the effects of deletion analysis in LMH cells, strongly argues that the only HNF3 recognition sequence required in order to activate the DHBV $\mathrm{S}$ promoter in these assays is the one at positions -25 to -36 . It is perhaps interesting that this location is normally where one would expect to find the TATA motif.

Experiments from our laboratory have also indicated in vitro binding sites for Sp1 downstream of the HNF3 site in the DHBV S promoter (unpublished observations), though it remains to be determined to what degree they are functional and what role they may play in coordinating activity from this promoter. As mentioned previously, Sp1 is capable of activating the HBV $S$ promoter through several functional recognition sites in the $S$ regulatory region (21), so it would not be unexpected if DHBV were able to make use of this transcription factor in regulating expression from the $\mathrm{S}$ promoter. However, an interesting difference from HBV is the stringent requirement that the DHBV S promoter displays for differentiated hepatocyte cell lines (17; this study), whereas the HBV $\mathrm{S}$ promoter is active in both HeLa and HepG2.1 cells as well as other nonliver cell lines $(4,11,21,22,25)$. Our observation that the DHBV S promoter did not activate expression of CAT in HeLa cells is consistent with a previous indication that the DHBV envelope promoters may not be functional in HeLa cells (12). Clearly, if ubiquitous transcriptional regulators, such as $\mathrm{Sp} 1$, have a role regulating transcription from this promoter, they are not capable of acting independently to promote transcription as is the case for the HBV S promoter (21).

\section{ACKNOWLEDGMENTS}

We are grateful to Alan McLachlan, The Scripps Research Institute, La Jolla, Calif., for the HepG2.1 cell line, and to Robert Costa, University of Illinois, Chicago, for plasmids pCMVHNF3 $\alpha$ and $\mathrm{pCM}$ VHNF3 $\beta$

T.W. thanks Alan McLachlan for numerous helpful discussions during this work

\section{REFERENCES}

1. Ausubel, F. M., R. Brent, R. E. Kingston, D. D. Moore, J. G. Seidman, J. A Smith, and K. Struhl. 1987. Current protocols in molecular biology. John Wiley \& Sons, New York.

2. Beg, A. A., T. S. Finco, P. V. Nantermet, and A. S. Baldwin, Jr. 1993. Tumor necrosis factor and interleukin-1 lead to phosphorylation and loss of IкB $\alpha$ : a mechanism for NF-кB activation. Mol. Cell. Biol. 13:3301-3310.

3. Buscher, M., W. Reiser, H. Will, and H. Schaller. 1985. Transcripts and the putative RNA pregenome of duck hepatitis B virus: implications for reverse transcription. Cell 40:717-724.

4. Chang, H.-K., and L.-P. Ting. 1989. The surface gene promoter of the human hepatitis B virus displays a preference for differentiated hepatocytes. Virology 170:176-183. 
5. Chang, H.-K., B.-Y. Wang, C.-H. Yuh, C.-L. Wei, and L.-P. Ting, 1989. A liver-specific nuclear factor interacts with the promoter region of the large surface protein gene of human hepatitis B virus. Mol. Cell. Biol. 9:51895197.

6. Condreay, L. D., C. E. Aldrich, L. Coates, W. S. Mason, and T. T. Wu. 1990. Efficient duck hepatitis B virus production by an avian liver tumor cell line. J. Virol. 64:3249-3258.

7. Costa, R. H., D. R. Grayson, and J. E. Darnell, Jr. 1989. Multiple hepatocyte-enriched nuclear factors function in the regulation of transthyretin and $\alpha 1$-antitrypsin genes. Mol. Cell. Biol. 9:1415-1425.

8. Crescenzo-Chaigne, B., J. Pillot, and A. Lilienbaum. 1995. Interplay between a new HNF3 and the HNF1 transcriptional factors in the duck hepatitis B virus enhancer. Virology 213:231-240.

9. Crescenzo-Chaigne, B., J. Pillot, A. Lilienbaum, M. Levrero, and E. Elfassi. 1991. Identification of a strong enhancer element upstream from the pregenomic RNA start site of the duck hepatitis B virus genome. J. Virol. 65:3882-3886.

10. Cullen, B. R., P. T. Lomedico, and G. Ju. 1984. Transcriptional interference in avian retroviruses: implication for the promoter insertion model of leukemogenesis. Nature (London) 307:241-244.

11. Faktor, O., T. DeMedina, and Y. Shaul. 1988. Regulation of hepatitis B virus S gene promoter in transfected cell lines. Virology 162:362-368.

12. Huang, M., and J. Summers. 1994. pet, a small sequence distal to the pregenome cap site, is required for expression of the duck hepatitis B virus pregenome. J. Virol. 68:1564-1572.

13. Jackson, D. A., K. E. Rowader, K. Stevens, C. Jiang, P. Milos, and K. S. Zaret. 1993. Modulation of liver-specific transcription by interactions between hepatocyte nuclear factor 3 and nuclear factor 1 binding DNA in close apposition. Mol. Cell. Biol. 13:2401-2410.

14. Johnson, J. L., A. K. Raney, and A. McLachlan. 1995. Characterization of a functional hepatocyte nuclear factor 3 binding site in the hepatitis B virus nucleocapsid promoter. Virology 208:147-158.

15. Kawaguchi, T., K. Momura, Y. Hirayama, and T. Kitagawa. 1987. Establishment and characterization of a chicken hepatocellular carcinoma cell line,
LMH. Cancer Res. 47:4460-4464.

16. Lilienbaum, A., B. Crescenzo-Chaigne, A. A. Sall, J. Pillot, and E. Elfassi. 1993. Binding of nuclear factors to functional domains of the duck hepatitis B virus enhancer. J. Virol. 67:6192-6200.

17. Liu, C., L. D. Condreay, J. B. E. Burch, and W. Mason. 1991. Characterization of the core promoter and enhancer of duck hepatitis B virus. Virology 184:242-252.

18. Liu, C., W. S. Mason, and J. B. E. Burch. 1994. Identification of factorbinding sites in the duck hepatitis B virus enhancer and in vivo effects of enhancer mutations. J. Virol. 68:2286-2296.

19. Mandart, E., A. Kay, and F. Galibert. 1984. Nucleotide sequence of a cloned duck hepatitis B virus genome: comparison with woodchuck and human hepatitis B virus sequences. J. Virol. 49:782-792.

20. Overdier, D. G., A. Porcella, and R. H. Costa. 1994. The DNA-binding specificity of the hepatocyte nuclear factor 3 /forkhead domain is influenced by amino acid residues adjacent to the recognition helix. Mol. Cell. Biol. 14:2755-2766.

21. Raney, A. K., H. B. Le, and A. McLachlan. 1992. Regulation of transcription from the hepatitis B virus major surface antigen promoter by the Sp1 transcription factor. J. Virol. 66:6912-6921.

22. Raney, A. K., D. R. Milich, A. J. Easton, and A. McLachlan. 1990. Differentiation-specific transcriptional regulation of the hepatitis B virus large surface antigen gene in human hepatoma cell lines. J. Virol. 64:2360-2368.

23. Raney, A. K., P. Zhang, and A. McLachlan. 1995. Regulation of transcription from the hepatitis B virus large surface antigen promoter by hepatocyte nuclear factor 3. J. Virol. 69:3265-3272.

24. Schneider, R., and H. Will. 1991. Regulatory sequences of duck hepatitis B virus $\mathrm{C}$ gene transcription. J. Virol. 65:5693-5701.

25. Standring, D. N., W. J. Rutter, H. E. Varmus, and D. Ganem. 1984. Transcription of the hepatitis B surface antigen gene in cultured murine cells initiates within the presurface region. J. Virol. 50:563-571.

26. Xanthopoulos, K. G., and J. Mirkovitch. 1993. Review: gene regulation in rodent hepatocytes during development, differentiation and disease. Eur. J. Biochem. 216:353-360. 